

\title{
Modeling laser drilling in percussion regime using constraint natural element method
}

Jérémie Girardot, Philippe Lorong, Lounès Illoul, Nicolas Ranc, Matthieu Schneider, Véronique Favier

\section{- To cite this version:}

Jérémie Girardot, Philippe Lorong, Lounès Illoul, Nicolas Ranc, Matthieu Schneider, et al.. Modeling laser drilling in percussion regime using constraint natural element method. International Journal of Material Forming, 2015, 15 p. 10.1007/s12289-015-1269-0 . hal-01285198

\section{HAL Id: hal-01285198 \\ https://hal.science/hal-01285198}

Submitted on 8 Mar 2016

HAL is a multi-disciplinary open access archive for the deposit and dissemination of scientific research documents, whether they are published or not. The documents may come from teaching and research institutions in France or abroad, or from public or private research centers.
L'archive ouverte pluridisciplinaire HAL, est destinée au dépôt et à la diffusion de documents scientifiques de niveau recherche, publiés ou non, émanant des établissements d'enseignement et de recherche français ou étrangers, des laboratoires publics ou privés. 


\title{
Modeling laser drilling in percussion regime using constraint natural element method
}

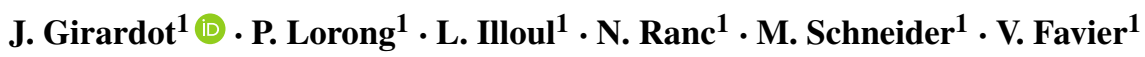

\begin{abstract}
The laser drilling process is the main process used in machining procedures on aeronautic engines, especially in the cooling parts. The industrial problematic is to reduce geometrical deviations of the holes and defects during manufacturing. The interaction between a laser beam and an absorbent metallic matter in the laser drilling regime involves thermal and hydrodynamical phenomenon. Their role on the drilling is not yet completely understood and a realistic simulation of the process could contribute to a better understanding of these phenomenon. The simulation of such process induces strong numerical difficulties. This work presents a physical model combined with the use of the original Constraint Natural Element Method to simulate the laser drilling. The physical model includes solid/liquid and liquid/vapor phase transformations, the liquid ejection and the convective and conductive thermal exchanges. It is the first time that all these phenomena are included in a modelling and numerically solved in a $2 \mathrm{D}$ axisymmmetric problem. Simulations results predict most of measurements (hole geometry, velocity of the liquid ejection and laser drilling velocity) without adjusting any parameters.
\end{abstract}

Keywords Drilling - Laser · Percussion regime · Simulation $\cdot$ CNEM $\cdot$ Phase Change

J. Girardot

jeremie.girardot@ensam.eu

1 Arts et Métiers Paristech / CNRS / CNAM, PIMM Laboratory, 151 Boulevard de l'hôpital, F-75013, Paris, France

\section{Introduction}

Parts located in the high pressure stage in aircraft engines are designed such that a cooling process is done thanks to several holes among structures like turbine blades or combustors (Figs. 1a and 1b). These holes are currently produced using mainly laser drilling. To increase the cooling performance, drilled parts are also coated with a thermal barrier [18]. The coated material is a thermal-refractory ceramic with a thickness around $200 \mu \mathrm{m}$. The presence of this coated before the drilling operation induces several defaults such as a macro delamination of the coatings [12] [15]. The holes are more or less inclined (up to $20^{\circ}$ from surface) and are millimetric. A good quality for the holes means that their diameter is uniform along the hole and no micro-structural defaults exists across the hole. Obtaining such holes remains challenging from an industrial point of view. Indeed, the thermal stresses during the laser drilling process create several defaults in the hole vicinity and especially in the melted area such as Heat Affected Zone (HAZ) and micro-cracks. It has been shown that such damages in the resulting structure decreases the mechanical properties [19]. Using numerical simulations is a great interest to predict the occurance and location of such damages.

A percussion regime is set with the laser drilling process. It involves a range of laser incident intensity from 5 to $20 \mathrm{MW} / \mathrm{cm}^{2}$ with a pulse duration from 0.1 to $1 \mathrm{~ms}$. This work deals only with the static beam configuration and not with a mobile source (the configuration is then called "percussion"). For this considered regime, the main interaction between the focused laser beam and the substrate consists in heating the surface up to high temperature. The heating induces fusion and vaporization of the material. The melt part is then ejected by the vapor pressure, creating a keyhole with vapor plume at the center. This interaction 
Fig. 1 Typical aircraft engine parts design

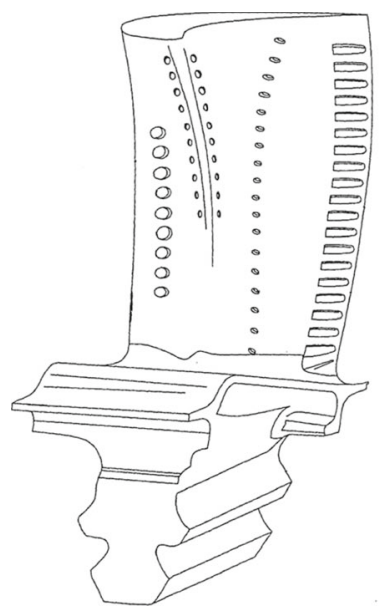

(a) Fan engine [5],

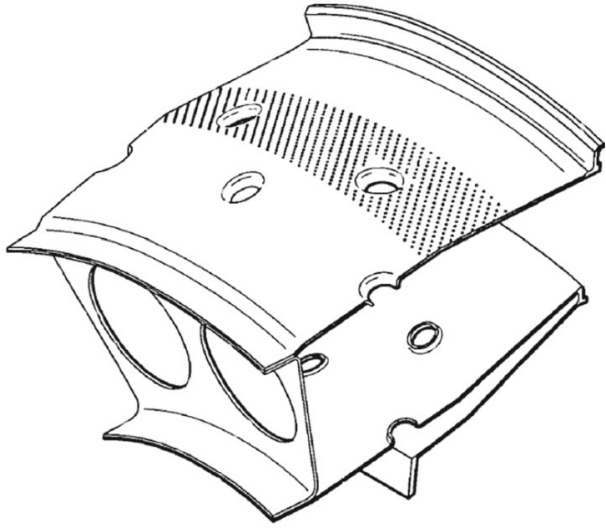

(b) Part of a combustor [6], acts all along the laser pulse leading to the formation of a hole. Figure 2 shows the physical phenomena involved during a laser drilling in the percussion regime. Many thermal exchanges between the laser beam, the moving liquid part and the solid part have to be taken into account in a simulation work.

The earliest modeling of laser drilling process was analytical [2]. This model contains all physical processes described previously and was used for the laser welding in [33], for the laser cutting [16] and for the laser drilling in [5, 23] and [31]. The output of this 1D model are the drilling velocity, the ejection melt velocity and the surface temperature but only at a steady state. The model is based on the momentum and energy equations written for a 1D domain, coupled with the vaporization law describing the behavior at the vapor front. Details about this law can be found in $[3,21]$ and [27]. Some extensions of the 1D basic analytical model were proposed to catch the change in time and space of these variables. However, strong hypotheses were introduced to cope with the numerical issues related to the fast phase transformations and high thermal gradient involved in laser processes. The works in [20] and [11] incorporated an analytical velocity profile to describe the melted flow. In [26], a pressure profile is assumed to reduce the free surface instabilities.

The basic finite element method is not well suitable for large deformation, strong field gradients and moving boundary problems. In this work, the Constraint Natural Element Method (CNEM) [37] is used as a numerical alternative method for laser drilling simulations. The CNEM is an extension of the Natural Element Method created and developed in [7, 35] and [34]. This method was developed to numerically solve non-convex and discontinuous problems.

The main purpose of the present paper is to solve the physical laser drilling model in order to catch the hole evolution in time and space. The use of the CNEM will allow us to deal with all the numerical issues described in the previous paragraph. Section "Physical model" describes the physical phenomena involved during laser drilling and explained the hypotheses chosen for modelling. Section "Numerical implementation" clarifies the implementation of the model from a numerical point of view. The setting up of an outflow boundary condition in the numerical model is also detailed. Section "Laser drilling simulation" presents the results of
Fig. 2 Physical processes during a laser drilling in the percussion regime

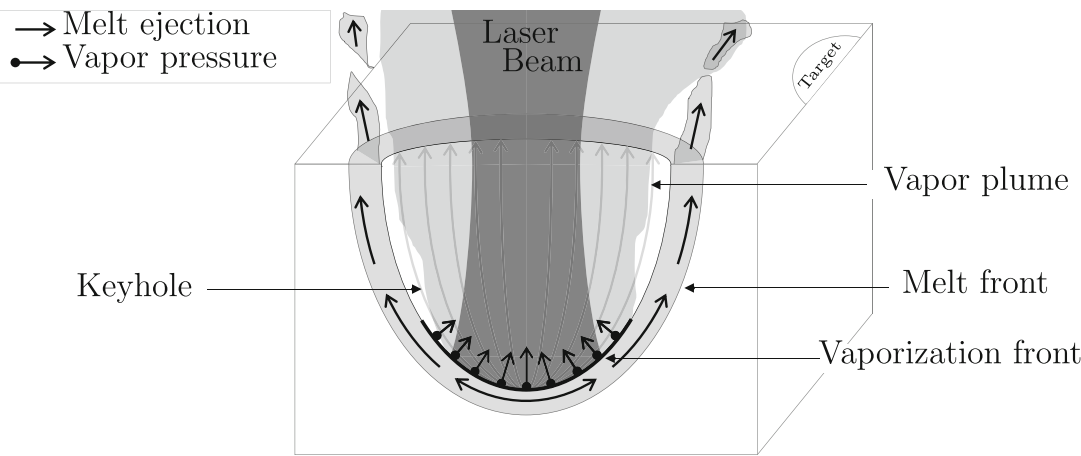


laser drilling simulation carried out on pure iron. The predicted results are discussed and compared with experimental ones.

\section{Physical model}

\section{Hypothesis}

The present model is based on the same equations and hypothesis as for the analytic model of [33]. The computation domain is assumed to be $2 \mathrm{D}$ with an axial symmetry such as the model would be able to represent only vertical drilling. Figure 3 shows the calculation domain in its axisymmetric frame $(\vec{r}, \vec{z})$ and its boundaries. A laser beam along the $\mathrm{z}$ axis is applied on the vaporization front. The domain considered for the simulation is about two or three times higher and stronger more than the laser beam radius, noted $r_{\text {laser }}$, in order to avoid side effects. The melted zone is delimited by the vapor and melt fronts. These two interfaces are moving interfaces. The energy conservation is solved in the solid and the liquid part where the thermal diffusion, phase changes (melting and boiling) and laser absorption are taken into account. The momentum conservation equation is only solved in the liquid zone and the liquid ejection outside the hole is not represented as we assume it does not play any role in the hole creation during the laser pulse. For the sake of simplicity, the vapor part is not described in the model. The vapor pressure, which induces the liquid ejection, is thus set only on the vaporization front free surface (see "Pressure on on the free surface" section). The mass loss by vaporization is also neglected as it appears during the numerical development that it does not influence significantly the vapor front position. This non-influence hypothesis was also assumed in [10] which simulated a laser drilling with greater laser intensity (around $100 \mathrm{MW} / \mathrm{cm}^{2}$ ) than in the present paper. It must be notted that the vapor phase is all neglected in the model except at the vapor front. Thus, physical effects induced by the vapor phase as a beam propagation medium, a phase flow or a heat source [4] cannot be described here. In their review, Schulz et al. [32] explain that the role of the vapor increases as the drilling is deep. As a consequence,
Fig. 3 Description of the simulated domain

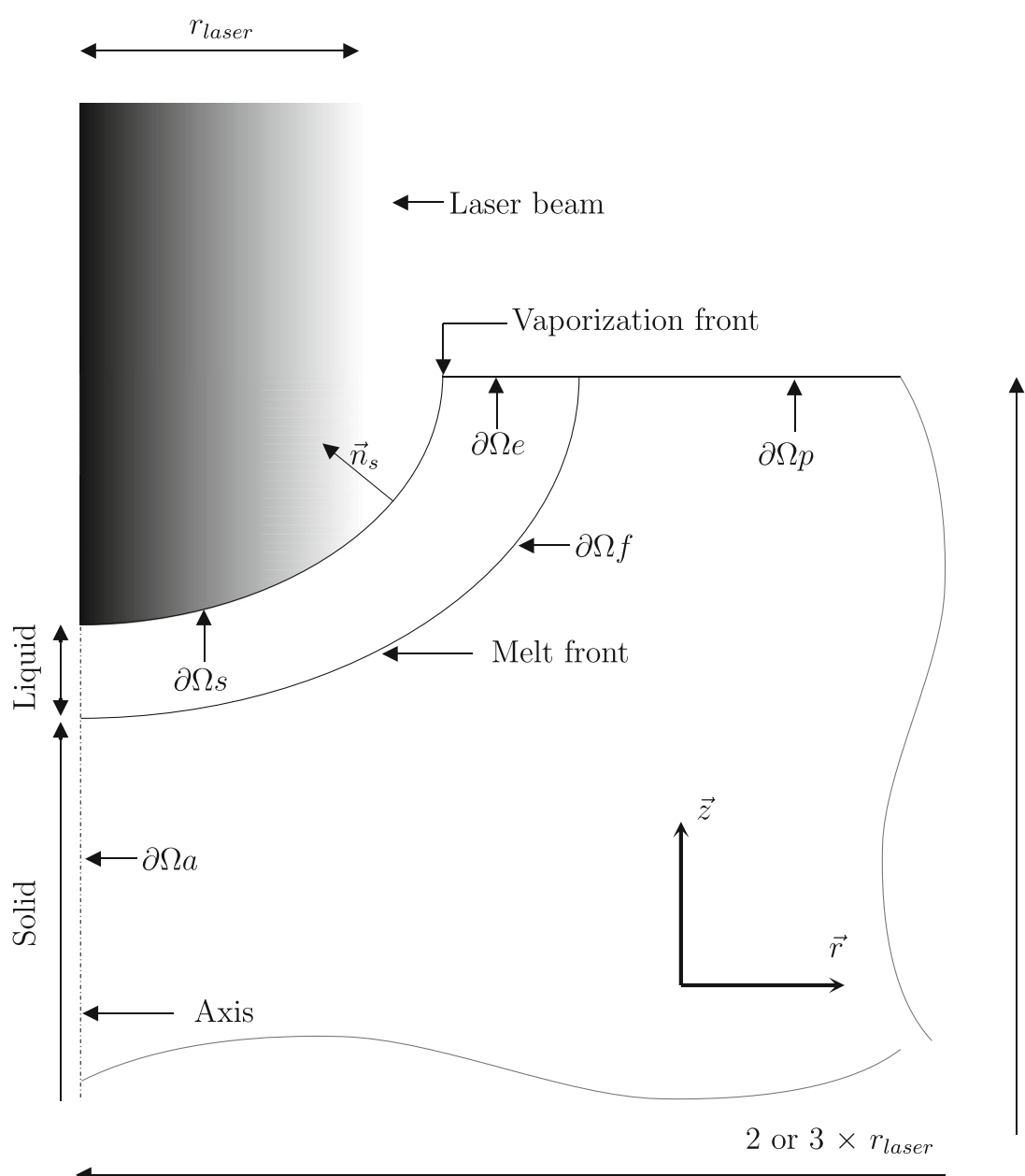


the present model will be only deal with small aspect ratio of the drill hole. The gravity is also not considered.

The axial symmetric space coordinate system is noted $(r, z)$, and the time is noted $t$. The unknowns in the model are the following:

- $\quad$ the temperature field noted $T(r, z, t)$;

- the volume enthalpy field noted $H(r, z, t)$;

- the velocity field noted $\vec{u}(r, z, t)$;

- the pressure field noted $p(r, z, t)$.

\section{Thermal problem}

The energy conservation equation is written in a lagrangian formulation $\left(\frac{\mathrm{d}}{\mathrm{d} t}\right.$ is the total derivative $)$ in both the liquid and solid zones with an enthalpy formulation :

$\frac{\mathrm{d} H}{\mathrm{~d} t}=-\operatorname{div}(\vec{q})$,

where $\vec{q}$ is the heat flux which is given by the Fourier law as a function the temperature :

$\vec{q}=-k \overrightarrow{\nabla T}$.

$k$ is the thermal conductivity in the considered medium (either solid or liquid). Based on the previous equation, a classical weak formulation is set and the volume integration of Eq. 1, considering a test function $T^{*}$, is written:

$\int_{\Omega} T^{*} \frac{\mathrm{d} H}{\mathrm{~d} t} \mathrm{~d} V=-\int_{\Omega} k \overrightarrow{\nabla T^{*}} \cdot \overrightarrow{\nabla T} \mathrm{~d} V+\int_{\partial \Omega} k T^{*} \overrightarrow{\nabla T} \cdot \overrightarrow{\mathrm{d} S}$,

where $\Omega$ is the considered volume and $\partial \Omega$ is its boundary, composed of $\partial \Omega_{s}, \partial \Omega_{e}, \partial \Omega_{p}$ and $\partial \Omega_{a}$.

The enthalpy/temperature law, noted $H(T)$, is chosen as a piecewise linear law:

$H(T)= \begin{cases}\rho_{s} C_{p}^{s} T & \forall T<T_{\text {sol }}, \\ \rho_{l} C_{p}^{l}\left(T-T_{l i q}\right)+\rho_{l} L_{m}+\rho_{s} C_{p}^{s} T_{\text {sol }} & \forall T>T_{\text {liq }},\end{cases}$

where $\rho_{s}$ and $\rho_{l}$ are the solid and liquid density, $C_{p}^{s}$ and $C_{p}^{l}$ are the solid and liquid heat capacity, respectively. $T_{s o l}$ and $T_{l i q}$ are respectively the solidus and liquidus temperatures, centered at the melting point. The interval temperature is chosen equal to $200 \mathrm{~K}$ and a piecewise linear function links the values $H\left(T_{s o l}\right)$ and $H\left(T_{l i q}\right)$. Figure 4 shows the enthalpy $H$ as a function of the temperature $T$ for pure iron.

In the thermal problem, all physical parameters (thermal conductivity and density) are distinct and constant regardless the temperature in the liquid and solid zones. A Heaviside type function is used to make a smooth transition near the boiling point.



Fig. 4 Enthalpy as a function of temperature (pure iron)

An adiabatic condition is set to the boundaries $\partial \Omega_{p}$ and $\partial \Omega_{a}$. The boundary $\partial \Omega_{e}$ represents an outflow condition such that the ejected fluid outside the hole is not simulated. As the model description is Lagrangian, a specific boundary condition is applied on $\partial \Omega_{e}$ and will be detailed in "Outflow boundary condition in a Lagrarian description" section of this paper. Boundary conditions on the free surface $\partial \Omega_{s}$ are described in the following sub-sections.

Intake Heat Flux on the Free Surface $\partial \Omega_{s}$

During the laser drilling, the intake heat flux is brought by the absorbed laser beam energy. This energy is modeled by a thermal flux, noted $\overrightarrow{\varphi \text { laser }}$, which corresponds to the laser peak power $P_{\text {peak }}$ divided by laser beam section and multiplied by three coefficients which takes into account:

- the spatial repartition of the laser source which is a function of the r position, noted $f(r)$;

- the time evolution of the laser pulse which is a function of time, noted $g(t)$;

- a laser absorption coefficient noted A.

Absorption in a keyhole can vary in a very complicated way, regarding the surface temperature [9] or hydrodynamic instabilities on the free surface [28]. In order to avoid the modelling of these complex effects, the absorption coefficients measured in [30] is used for several laser peak power corresponding to the percussion regime (Fig. 5a). Finally, the expression of $\overrightarrow{\varphi_{\text {laser }}}$ is :

$\overrightarrow{\varphi_{\text {laser }}}=-A f(r) g(t) \frac{P_{\text {peak }}}{\pi r_{\text {laser }}^{2}} \vec{z}$,

where:

- $g(t)$ ranges between 0 and 1 and is composed of two exponential functions, the first is increasing and the 
Fig. 5 Absorption coefficients and laser source functions



(a) Absorption $A$ from [28],

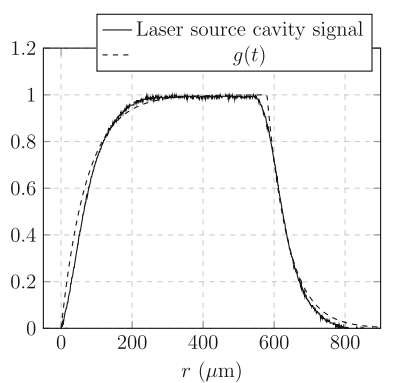

(b) Temporal shape $g(t)$,



(c) Spatial shape $f(r)$, second is decreasing. Their parameters are set to fit measurements done on the laser source cavity (Fig. 5b):

$$
g(t)= \begin{cases}0 & \text { if } t<0, \\ 1-\exp \left(\frac{-t}{\tau_{u p}}\right) & \text { if } 0 \leq t \leq t_{\text {pulse }}, \\ 1-\exp \left(\frac{-\left(t-t_{\text {pulse }}\right)}{\tau_{\text {down }}}\right) & \text { if } t>t_{\text {pulse }},\end{cases}
$$

where $t_{\text {pulse }}$ is the pulse duration and $\tau_{u p}$ and $\tau_{\text {down }}$ are the fitting parameters ;

- $f(r)$ ranges also between 0 and 1, and is a hyperGaussian function (also called top-hat) and ranges between 0 and 1 (Fig. 5c):

$$
f(r)=\exp \left(\frac{-r^{N}}{2\left(\frac{R_{f}}{1.177}\right)}\right),
$$

where $N$ and $R_{f}$ are the shape parameters of the function (if $N$ equals $2, f(r)$ is a gaussian function).

\section{Outtake heat flux on the free surface $\partial \Omega_{s}$}

The outtake heat flux on the free surface corresponds to the energy loss by vaporization which is the product between the vapor latent heat noted $L_{v}$ and the vaporized mass flow rate noted $\dot{M}_{v}$.

The mass flow rate is given by the Hertz-Langmuir relation obtained by writing the mass flow conservation equation at the vapor front [3]. The Hertz-Langmuir equation is a function of the surface temperature $T_{s}$ :

$\dot{M}_{v}\left(T_{s}\right)=\sqrt{\frac{m}{2 \pi k_{b}}} \frac{P_{s a t}\left(T_{s}\right)}{\sqrt{T_{s}}}\left(1-\beta_{r}\right)$,

where $m$ is the atomic mass, $k_{b}$ the Boltzmann constant and $P_{\text {sat }}$ is the vapor pressure given by the Clapeyron relation :

$P_{\text {sat }}\left(T_{s}\right)=P_{a t m} \exp \left(\frac{M_{a} L_{v}}{R}\left(\frac{1}{T_{v}}-\frac{1}{T_{s}}\right)\right)$,

with $M_{a}$ the molar mass, $R$ the ideal gas constant and $T_{v}$ the boiling temperature at room pressure.
The $\beta_{r}$ coefficient, ranging between 0 and 1 , represents retro-diffusion mechanisms which occurs at an evaporating surface ([22] and [36]). This mechanism consists on the recondensation (or retro-diffusion) of a portion of vapored matter. Figure 6 shows values of $\dot{M}_{v}$ for the three different values of $\beta_{r}$ :

- $\beta_{r}=1$ corresponds to the case for which all the vaporized matter is re-condensed such that $\dot{M}_{v}$ is null ;

- $\beta_{r}=0$ corresponds to the no-recondensation case ;

- $\beta_{r}=0.17$, is the maximum value in the case of a supersonic vaporized gas flow [27] which is the case during a laser drilling.

Therefore the Hertz-Langmuir equation describes the vaporization at the free surface and sets a non-null value for $\dot{M}_{v}$ before the boiling point temperature (Fig. 6). This is contradictory to the study led in [16]. Indeed, this work showed that when the surface temperature was lower than the boiling point, no movement of the melt zone was observed and no vaporization occured. In order to have the same effect in our model, a fit curve based on a spline function is built and represented on the Fig. 6 ("fit" curve). This curve is set on 0 for temperature lower than $T_{v}$ and then converges to the Hertz-Langmuir law with a $\beta_{r}$ equal to 0.17 .



Fig. 6 Vaporized mass flow rate as a function of the surface temperature (for pure iron) 
Finally, the boundary condition on the free surface $\partial \Omega_{s}$ is written:

$k \overrightarrow{\nabla T} \cdot \overrightarrow{\mathrm{d} S}=\overrightarrow{\varphi_{\text {laser }}} \cdot \overrightarrow{n_{s}}-L_{v} \dot{M}_{v}\left(T_{S}\right)$.

\section{Fluid Problem}

The fluid mechanics equations are only solved in the liquid zone (colored part in Fig. 3) as we assumed the velocity in the solid zone is null. The system of equations is composed of the momentum and mass conservation (fluid is assumed incompressible) plus constitutive equation for the liquid, assumed to be a Newtonian fluid :

$$
\left\{\begin{aligned}
\rho_{l} \frac{\mathrm{d} \vec{u}}{\mathrm{~d} t} & =\overrightarrow{\operatorname{div}(\overrightarrow{\bar{\sigma}})}, \\
\operatorname{div}(\vec{u}) & =0, \\
\overline{\bar{\sigma}} & =-p \overline{\bar{\delta}}+2 \mu \overline{\bar{D}},
\end{aligned}\right.
$$

with:

$\overline{\bar{D}}=\left(\begin{array}{cc}\frac{\partial u}{\partial r} & \frac{1}{2}\left(\frac{\partial u}{\partial z}+\frac{\partial v}{\partial r}\right) \\ \frac{1}{2}\left(\frac{\partial u}{\partial z}+\frac{\partial v}{\partial r}\right) & \frac{\partial v}{\partial z}\end{array}\right)$

where $\mu$ is the dynamic viscosity and $\rho_{l}$ is the liquid density.

A classical weak formulation is set and the volume integration of Eq. 11, considering test functions $u^{*}$ and $p^{*}$, is written:

$\left\{\begin{array}{l}\int_{\Omega} \rho_{l} \vec{u}^{*} \frac{\mathrm{d} \vec{u}}{\mathrm{~d} t} \mathrm{~d} V=-\int_{\Omega} \nabla \vec{u}^{*}: \overline{\bar{\sigma}} \mathrm{d} V+\int_{\partial \Omega} \vec{u}^{*} \cdot \overline{\bar{\sigma}} \cdot \overrightarrow{\mathrm{d} S}, \\ \int_{\Omega} p^{*} \operatorname{tr}(\overline{\bar{D}}) \mathrm{d} V=0 .\end{array}\right.$

A slip condition is set on the axis boundary $\partial \Omega_{a}$ :

$\vec{u} \cdot \vec{r}=0$

and a non-slip condition is applied on the melt front $\partial \Omega_{f}$.

$\vec{u}=\overrightarrow{0}$.

For the same reasons as in the thermal problem, boundary on $\partial \Omega_{e}$ cannot be an outflow condition because of the Lagrangian description. Specific treatments for this boundary are described in "Outflow boundary condition in a Lagrangian description" section.

\section{Pressure on the free surface}

A normale pressure $P_{s}$ is applied on the free surface $\partial \Omega_{s}$ which is the vapor pressure and is proportional to the vapor pressure $P_{\text {sat }}\left(T_{S}\right)($ Eq. 9):

$P_{s}\left(T_{s}\right)=\frac{P_{s a t}\left(T_{s}\right)}{2}\left(1+\beta_{r}\right)$

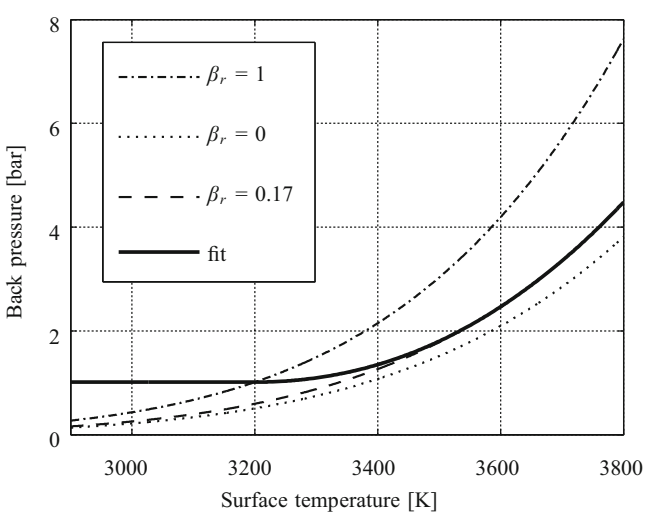

Fig. 7 Surface pressure as a function of temperature (for pure iron)

The retro-diffusion effect leads also to the presence of the $\beta_{r}$ coefficient. For the same reasons as for the Hertz-Langmuir expression, a "fit" function is used in our model to put the pressure equal to the room pressure for temperatures lower than the boiling temperature $T_{v}$ (Fig. 7).

During the laser drilling, the liquid free surface is subjected to surface tension pressures, noted $P_{\sigma}$. It is set as an additional boundary condition on $\partial \Omega_{s}$ :

$P_{\sigma}=\gamma\left(\frac{1}{R_{1}}+\frac{1}{R_{2}}\right)$,

where $\gamma$ is the surface tension coefficient which is assumed constant with temperature. $R_{1}$ and $R_{2}$ are primary and secondary curvatures of the free surface. $R_{1}$ is directly obtained by computing the curvature in the $(r, z)$ frame. $R_{2}$ is obtained by taking into account the axial symmetry:

$R_{2}(r, z)=\frac{r}{\left(\frac{\mathrm{d} z}{\mathrm{~d} r}\right)} \sqrt{1+\left(\frac{\mathrm{d} z}{\mathrm{~d} r}\right)^{2}}$.

Finally, the oundary condition on $\partial \Omega_{s}$ is written:

$\overline{\bar{\sigma}} \cdot \overrightarrow{\mathrm{d} S}=\left(P_{S}\left(T_{S}\right)+P_{\sigma}\right) \cdot \overrightarrow{n_{s}}$.

\section{Outflow boundary condition in a Lagrangian description}

The Lagrangian formulation is chosen to write heat and fluid equations. It induces a specific treatment of the boundary $\partial \Omega_{e}$. This boundary corresponds to the removal melted matter and has to be similar to a typical outflow boundary condition in an Eulerian description. In [10], a simple free boundary condition is set which is theorically wrong, but does not induces instabilities as the pulse duration is down to 200ns. It was observed that such boundary condition leads to unacceptable instabilities in the melted zone for the percussion regime. In order to avoid it, it is proposed here to set a Neumann boundary condition for the 
Fig. 8 Scheme of the cutting boundary condition

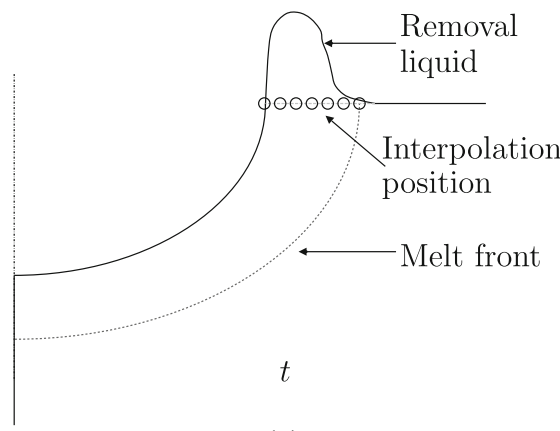

(a)

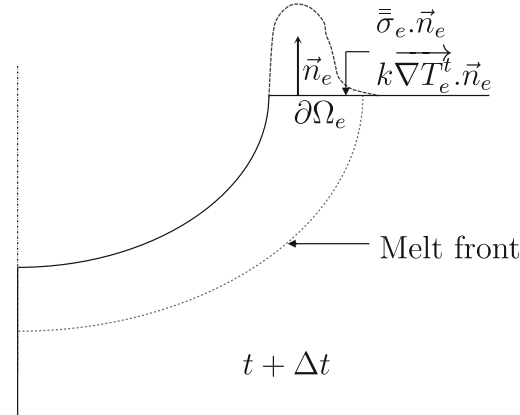

(b) heat and fluid problem which described the energetic state of the liquid just before cutting it at time $t$ (Fig. 8a). An interpolation operation gives temperature, velocity and pressure state at the cutting position, noted $T_{e}, \overrightarrow{u_{e}}$ and $p_{e}$. The boundary condition applied to the new boundary $\partial \Omega_{e}$ at time $t+\mathrm{d} t$ (Fig. 8b) is a heat flux for the thermal problem:

$k \overrightarrow{\nabla T} \cdot \overrightarrow{d S}=k \overrightarrow{\nabla T_{e}} \cdot \overrightarrow{n_{e}}$

and a force for the fluid problem:

$\left(-p_{e} \overline{\bar{\delta}}+2 \mu \overline{\bar{D}}\left(\overrightarrow{u_{e}}\right)\right) \cdot \overrightarrow{n_{e}}=\overline{\overline{\sigma_{e}}} \cdot \overrightarrow{n_{e}}$

As far as authors are aware, no such specific treatment was developed in Lagrangian simulation of a moving fluid so it needs to be validated (see "Validation of the boundary condition" section).

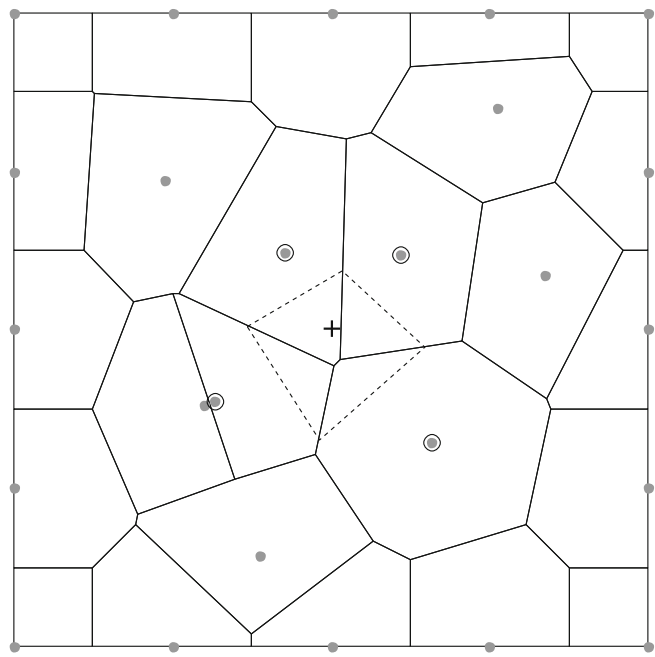

- Node

- Neighboors $+\quad x(r, z, t)$

Fig. 9 Natural neighbor concept, used to interpolate $f(r, z, t)$

\section{Numerical implementation}

\section{Space discretization using Constraint Natural Elements}

The physical model presented in the previous section is solved using the Constraint Natural Elements Method (CNEM). In the same way that the finite element method, the CNEM approximates a field by using a variational/weak formulation and uses an interpolation function built thanks to a Vorono diagram like the Sibson function ([37] and [8]). This method allows to work in Lagrangian description but with no mesh distortion problem. It already showed its capability to simulate the behavior of a liquid free surface [14] and a moving melting front interface [38]. More recently the method was extended to 3D simulations [17].

The physical model presented in the previous section is solved using the Constrained Natural Elements Method (CNEM). The CNEM comes from the Natural Element Method (NEM) [34]. The NEM is based, as for the finite element method, on the Galerkin method. However, the NEM uses the Natural Neighbor Interpolation inside the variational/weak formulation. The Natural Neighbor Interpolation is built thanks to a Vorono diagram like the Sibson function ([37] and [8]). This kind of interpolation allows working in updated Lagrangian descriptions but with no mesh distortion problem [7]. The specificity of the CNEM is the usage of the constrained Vorono diagram (dual of the constrained Delaunay tessellation) to compute the Natural Neighbor functions. It allows dealing with strongly non-convex domain by introducing a geometric description of the boundary of the domain. The use of a-shapes with the NEM sidesteps this description and showed its capability to simulate the behavior of a liquid free surface [14] or for the simulation of laser surface coating processes [13]. Nevertheless when local non-convexities on the boundary are observed, or when huge gradients inside the node distribution are present is not easy to apply a-shapes without creating non-realistic voids inside the domain or on its boundary. In the same idea, it is not easy to introduce discontinuities along non-convex interfaces inside the domain 
with a-shapes. Nevertheless there is also a drawback with the CNEM as it is necessary to maintain, during the simulation, a consistent geometric description of interfaces and boundaries. If it is not complex to manage it in $2 \mathrm{D}$ it will be significantly more complex in 3D. The CNEM was already used for moving melting front interface [38] and more recently extended to 3D simulations [17].

The volume integration is done at the node of the mesh. Thus, considering a field noted $f$ which is a function of the space and time system $\Omega(t)$, and $f_{i}$ the node value in the CNEM system, the approximation gives:

$\forall(r, z) \in \Omega(t), \quad f(r, z, t)=\sum_{i=1}^{V} f_{i}(t) \phi_{i}^{c}(r, z, t)$,

with $V$ the number of natural neighbors (Fig. 9) for the point $(r, z, t)$ and $\phi_{i}^{c}$ the constraint Sibson interpolation function associated to node $i$. The vector $\mathbf{F}$ is then composed of the nodal values of $f_{i}$.

\section{Time discretization in a Lagrangian description}

As the Lagrangian description is used, the nodes must follow the moving liquid zone. The node position update is done with an explicit first order Euler scheme:

$R \vec{r}+Z \vec{z}=r \vec{r}+z \vec{z}+\vec{u} \Delta t$,

where $(r, z)$ and $(R, Z)$ are respectively the node coordinates at time $t$ and $t+\Delta t$.

The time scheme used in this work is a first order implicit Euler scheme which gives, with a step time $\Delta t$, the time derivative of a field $f$ at the time $t$ :

$\left.\frac{\mathrm{d} f}{\mathrm{~d} t}\right|_{t} \approx \frac{f(R, Z, t+\Delta t)-f(r, z, t)}{\Delta t}$.

Temperature, enthalpy, velocity and pressure are integrated on nodes so their values are kept constant during the space actualization which makes the development easier and the calculation time lower by avoiding some interpolation operations.

\section{Systems of equations}

By substituting $H, T$ and $T^{*}$ in the weak formulation (Eq. 3), the following system of equations is obtained:

$[M] \frac{\mathbf{H}^{t+\Delta t}-\mathbf{H}^{t}}{\Delta t}+[K] \mathbf{T}^{t+\Delta t}=\mathbf{F}$,

where $\mathbf{T}$ and $\mathbf{H}$ are two $n \times 1$ vectors $(n$ is the number of nodes) containing nodal temperatures and enthalpies. Considering $\phi$ as the Sibson interpolation function linked to both of these fields, $[K],[M]$ and $\mathbf{F}$ are:

$$
\begin{aligned}
& \forall(i, j) \in \llbracket 1, n \mathbb{\rrbracket}^{2}, \\
& K_{i j}= \int_{\Omega_{n} k_{i} \overrightarrow{\nabla \phi_{i}} \cdot \overrightarrow{\nabla \phi_{j}} \mathrm{~d} V,} \\
& M_{i j}=\left\{\begin{array}{cc}
\int_{\Omega_{n}} \phi_{i} \phi_{j} \mathrm{~d} V & \text { if } i=j, \\
0 & \text { if } i \neq j,
\end{array}\right. \\
& \forall(i, j) \in \llbracket 1, n \rrbracket, F_{i}= \begin{cases}0 & \text { if } i \in \partial \Omega_{a}, \\
0 & \text { if } i \in \partial \Omega_{p}, \\
\int_{\partial \Omega_{s}}\left[\overrightarrow{\varphi_{\text {laser }} \cdot \vec{n}_{s}}-L_{v} \dot{M}_{v}\left(T_{i}\right)\right] \mathrm{d} S & \text { if } i \in \partial \Omega_{s}, \\
\int_{\partial \Omega_{e}}\left[k_{e} \overrightarrow{\nabla T_{e}^{t}} \cdot \overrightarrow{n_{e}}\right] \mathrm{d} S & \text { if } i \in \partial \Omega_{e} .\end{cases}
\end{aligned}
$$

In the same way, by substituting fields $\vec{u}, p, \vec{u}^{*}$ and $p^{*}$ in the weak formulation 13 , the following system of equations is obtained:

$\left[[M] \frac{1}{\Delta t}+[K]\right] \mathbf{U}^{t+\Delta t}=\left(\begin{array}{c}\mathbf{F}^{r} \\ \mathbf{F}^{z} \\ 0\end{array}\right)+[M] \frac{\mathbf{U}^{t}}{\Delta t}$,

where $\mathbf{U}$ is a $3 n \times 1$ vector containing nodal velocities in $r$ and $z$ directions and nodal pressures. The Sibson function $\phi$ is used to interpolate velocities and a Heaviside function $\psi$ assumed to be constant in each Voronoi cells
Fig. 10 Description of the liquid extraction using the melt front position

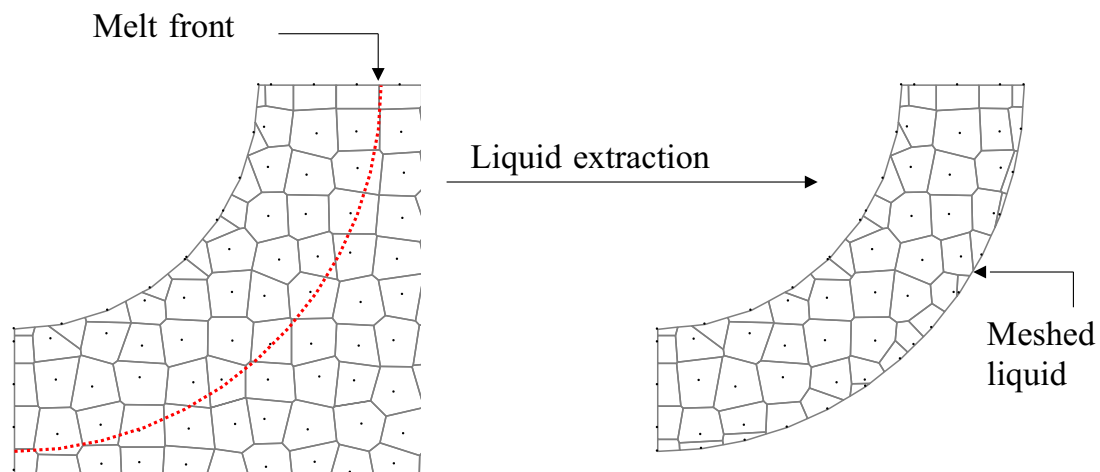

(a) 
is used to interpolate pressures, as following the work in [14].

$\forall(i, j) \in \llbracket 1, n \rrbracket^{2}$,

$K_{i j}=\int_{\Omega_{n}}\left[\begin{array}{lll}2 \mu\left(\phi_{i, x} \phi_{j, x}+\frac{\phi_{i, y} \phi_{j, y}}{2}+\frac{\phi_{i} \phi_{j}}{r^{2}}\right) & \mu \phi_{i, y} \phi_{j, x} & -\left(\phi_{i, x}+\frac{\phi_{i}}{r}\right) \psi_{j} \\ \mu \phi_{i, y} \phi_{j, x} & 2 \mu\left(\phi_{i, y} \phi_{j, y}+\frac{\phi_{i, x} \phi_{j, x}}{2}\right) & -\phi_{i, y} \psi_{j} \\ -\left(\phi_{j, x}+\frac{\phi_{j}}{r}\right) \psi_{i} & -\psi_{i} \phi_{j, y}\end{array}\right] \mathrm{dV}$

$M_{i j}=\left[\begin{array}{lll}\int_{\Omega_{n}} \phi_{i} \phi_{i} \mathrm{dV} & 0 & 0 \\ 0 & \int_{\Omega_{n}} \phi_{i} \phi_{i} \mathrm{dV} & 0 \\ 0 & 0 & \int_{\Omega_{n}} \phi_{i} \phi_{i} \mathrm{~d} V\end{array}\right]$

$\mathbf{F}^{r}$ and $\mathbf{F}^{z}$ are:

$\forall(i, j) \in \llbracket 1, n \rrbracket, F_{i}^{r}=\left\{\begin{array}{lr}0 & \text { if } i \in \partial \Omega_{a}, \\ 0 & \text { if } i \in \partial \Omega_{p}, \\ \int_{\partial \Omega_{s}}\left(\left(P_{s}\left(T_{i}\right)+P_{\sigma}\right) \cdot \overrightarrow{n_{s}} .\right) \cdot \vec{r} \mathrm{~d} S & \text { if } i \in \partial \Omega_{s}, \\ \int_{\partial \Omega_{e}}\left(\overline{\overline{\sigma_{e}^{t}}} \cdot \overrightarrow{n_{e}}\right) \cdot \vec{r} \mathrm{~d} S & \text { if } i \in \partial \Omega_{e} .\end{array}\right.$

$\forall(i, j) \in \llbracket 1, n \rrbracket, F_{i}^{z}=\left\{\begin{array}{lr}0 & \text { if } i \in \partial \Omega_{a}, \\ 0 & \text { if } i \in \partial \Omega_{p}, \\ \int_{\partial \Omega_{s}}\left(\left(P_{s}\left(T_{i}\right)+P_{\sigma}\right) \cdot \overrightarrow{n_{s}} \cdot\right) \cdot \vec{z} \mathrm{~d} S & \text { if } i \in \partial \Omega_{s}, \\ \int_{\partial \Omega_{e}}\left(\overline{\overline{\sigma_{e}}} \cdot \overrightarrow{n_{e}}\right) \cdot \vec{z} \mathrm{~d} S & \text { if } i \in \partial \Omega_{e} .\end{array}\right.$

\section{Incremental scheme}

The equation systems to solve at each time step are given in the previous section and are the main parts of the incremental scheme of the simulation. Some re-meshing operations are also required and especially dedicated to the removal of the nodes outside of the hole and to melt zone description:

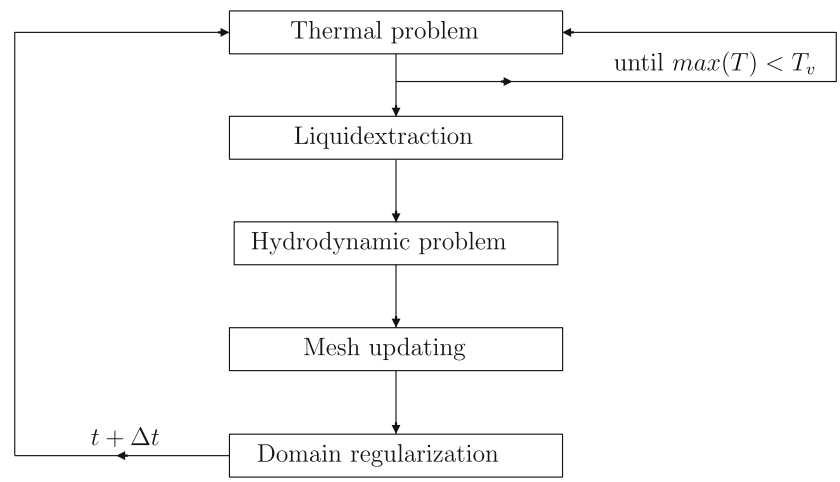

Fig. 11 Incremental scheme
$[K]$ and $[M]$ are two $3 n \times 3 n$ square matrix such that $K_{i j}$ and $M_{i j}$ are two $3 \times 3$ matrix linking the nodes $i$ and $j$ :

- the extraction of the liquid zone is done at each time step and consists in creating a new fluid domain with nodes whose the temperature is higher than the boiling point (Fig. 10). On the contrary of the work in [38] also using the CNEM, the moving melt interface is no more described with same nodes. New nodes are distributed along the isovalue $T_{m}$ at each time step and used to construct (i.e. meshed) the melt zone (Fig. 10). This operation is greatly simplified by the use of the CNEM;

- the cutting of the ejected zone is done in the same way than the liquid extraction, except that new nodes are distributed on top surface $z=0$ and not on an isovalue. A new domain is constructed (i.e. meshed) by omitting the ejected nodes (added nodes are illustrated in Fig. 8 by the interpolation positions).

- finally, a re-meshing operation is required to assure a satisfying nodal density in the liquid zone during the calculation. and also to manage the cutting of the ejected liquid zone.

All these re-meshing operations are located at the end of each increment scheme (Fig. 11), after computing temperature, velocity and pressure fields, and updating node positions. By re-meshing, we mean that some nodes are moved or added and the interpolation functions $\phi$ are recalculated. It is also noticed that the thermal problem is solved only before the surface temperature reaches the boiling point.

Table 1 Step time and mesh size for the simulations

\begin{tabular}{lll}
\hline Peak power $[\mathrm{kW}]$ & Mesh size $[\mu \mathrm{m}]$ & Step time $[\mathrm{ns}]$ \\
\hline 3 & 1 & 30 \\
5 & 1 & 20 \\
7 & 0.7 & 20 \\
10 & 0.5 & 10 \\
13 & 0.5 & 10 \\
\hline
\end{tabular}



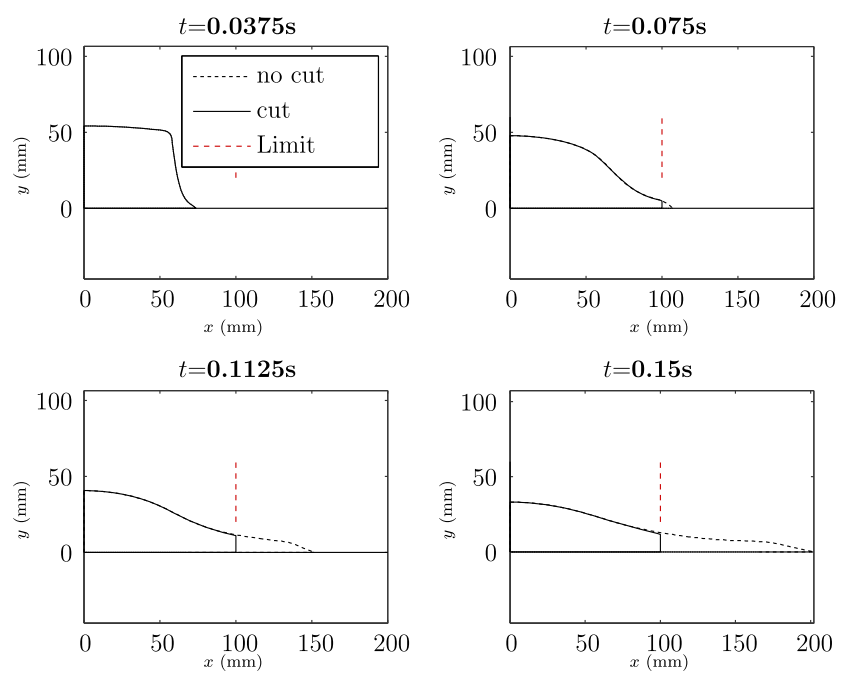

Fig. 12 Free surface positions obtained by numerical simulations with and without cutting in a broken dam problem

Through this incremental scheme, the coupling between thermal and fluid problem is solve segregatly (the thermal problem is solved before the fluid one during a step time). Thus, the step time, noted $\Delta t$, needs to be chosen carefully especially because of the exponential law linking the surface temperature and the surface pressure (Eq. 16). To avoid an unstable surface pressure, the step time needs to be sufficiently small. The Table 1 gives the step times used for the simulations. For a laser intensity range between 3 to $13 \mathrm{MW} / \mathrm{cm}^{2}$, the step time needs to be ranged between 30 to $10 \mathrm{~ns}$. The corresponding mesh size in the liquid zone, noted $h$, is obtained by the following stability

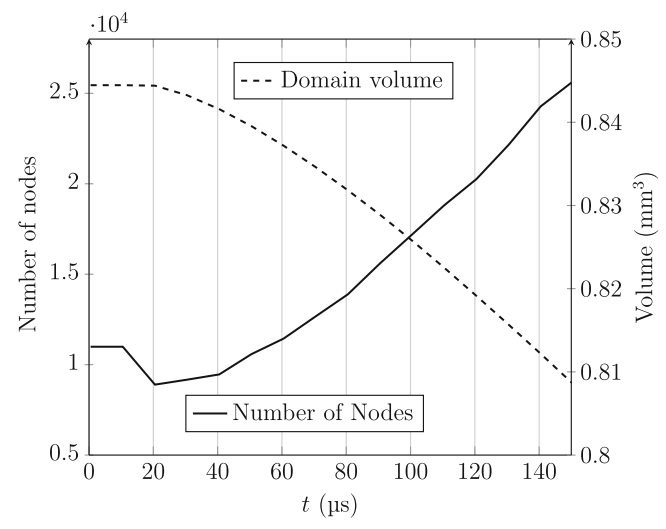

Fig. 13 Number of nodes and volume domain evolution during the simulation

equation:

$h \leq \sqrt{\frac{k_{l}}{\rho_{l} C_{p}^{l}} \Delta t .}$

\section{Validation of the boundary condition $\partial \Omega_{e}$}

The aim of this validation is to confront our model with a free surface fluid problem and to compare results with and without a cutting boundary limit. The chosen problem is the broken dam simulation already used in [14] to validate a model based on alpha-NEM method. The simulation is done in a 2D plane strain frame $(x, y)$ with an initial square domain of water subjected to gravity. The walls at the left and the bottom of the water domain are described with a perfect slip boundary condition. Only the fluid equation is
Table 2 Input parameters: thermophysical values from [25] and mesures from [30]

\begin{tabular}{lll}
\hline Solid density & $\rho_{s}$ & $7874 \mathrm{~kg} / \mathrm{m}^{3}$ \\
Liquid density & $\rho_{l}$ & $6980 \mathrm{~kg} / \mathrm{m}^{3}$ \\
Solid conductivity & $k_{s}$ & $70 \mathrm{~W} /(\mathrm{m} . \mathrm{K})$ \\
Liquid conductivity & $k_{l}$ & $38 \mathrm{~W} /(\mathrm{m} . \mathrm{K})$ \\
Solid heat capacity & $C_{p}^{s}$ & $450 \mathrm{~J} /(\mathrm{kg} \cdot \mathrm{K})$ \\
Liquid heat capacity & $C_{p}^{l}$ & $824 \mathrm{~J} /(\mathrm{kg} \cdot \mathrm{K})$ \\
Melting latent heat & $L_{m}$ & $272 \times 10^{3} \mathrm{~J} / \mathrm{kg}$ \\
Boiling latent heat & $L_{v}$ & $6095 \times 10^{3} \mathrm{~J} / \mathrm{kg}$ \\
Melting point & $T_{m}$ & $1800 \mathrm{~K}$ \\
Boiling point & $T_{v}$ & $3200 \mathrm{~K}$ \\
Dynamic viscosity & $\mu$ & $3.7 \times 10^{-3} \mathrm{~Pa} . \mathrm{s}$ \\
Surface tension coefficient & $\gamma$ & $1.8 \mathrm{~N} / \mathrm{m}$ \\
Molar mass & $M_{a}$ & $5.58 \times 10^{-2} \mathrm{~kg} / \mathrm{mol}$ \\
Atomic mass & $m$ & $9.3 \times 10^{-26} \mathrm{~kg}$ \\
Peak Power & $P_{p e a k}$ & $7 \mathrm{~kW}$ \\
Absorption coefficient (corresponding to $7 \mathrm{~kW})$ & $A$ & 0.68 \\
Laser beam radius (top-hat shape) & $r_{\text {laser }}$ & $150 \mu \mathrm{m}$ \\
\hline
\end{tabular}


Fig. 14 Drilling profile at 50, 100 and $150 \mathrm{~ms}$
(a) $50 \mu \mathrm{s}$

(b) $100 \mu \mathrm{s}$

(c) $150 \mu \mathrm{s}$

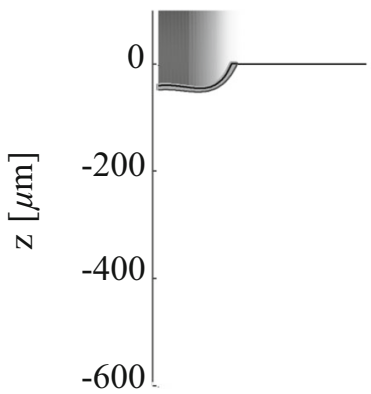

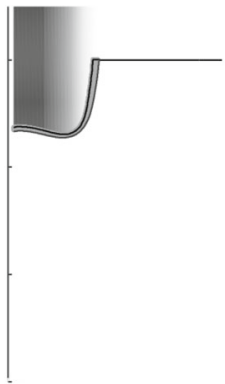

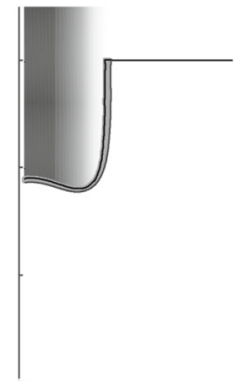

solved. No heat transfer is taken into account in this simulation. A first simulation is done with no additional conditions and results are in good agreements in terms of free surf ace position during the simulation regarding results found in [14]. The front domain position is also in the same tendancies than measures done in [24]. The second simulation is the same with an additional cutting boundary condition situated at $x=100 \mathrm{~mm}$. The liquid domain flows is subjected to its own weight on the right side until reaching the cutting limit. Concerning this simulation, the free surface stops evolving at the limit and remains at the same position until the end of the simulation. But the fluid flow allows to evacuate the liquid so the free surface position at the top is the same for both simulations.

Figure 12 shows the evolution of the free surface for simulations named "no cut" and "cut". The cutting limit is plotted with a vertical dashed line.

It is noticed that the management of an outflow boundary condition in a Lagrangian description does not induce in our model some instabilities or fluid flow modifications regarding the non-cutting condition.

It is noticed that other validations were made and are not detailed in this paper:

- validation of heat transfers in a moving fluid (convection);

- enthalpic formulation;
- surface vaporization heat transfers problem;

- surface tension implementation.

These validations are made by comparing numerical results with an analytical solution. For all validations cases, the relative error between numeric and analytic results never overtakes $3 \%$.

\section{Laser drilling simulation}

\section{Inputs}

Table 2 shows input parameters of the simulation. The thermo-physical properties correspond to pure iron and are assumed to be closed to the properties of low carbon steels. Every values of thermophysical properties were found in the litterature [25]. Only the absorption coefficient $(0.68)$ and the laser beam radius $(150 \mu \mathrm{m})$ come from experimental measurements done in [30]. The initial domain is a $400 \times 800 \mu m^{2}$ rectangular and the simulation duration is set to $150 \mu \mathrm{s}$. The end of the laser drilling during the cooling of the domain is not simulated. The laser incident intensity is equal to $10 \mathrm{MW} / \mathrm{cm}^{2}$. The parameters for spatial and temporal distributions are chosen to fit datas of the laser source (Trumpf HL201p) used in the present study.
Fig. 15 Temperature and drilling velocity during the simulation



(a) Drilling velocity $(\mathrm{m} / \mathrm{s})$,

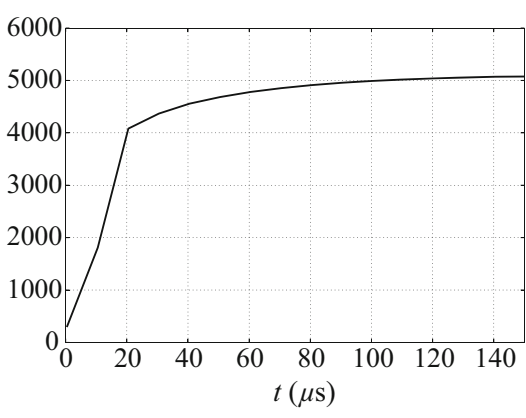

(b) Maximum temperature (K), 


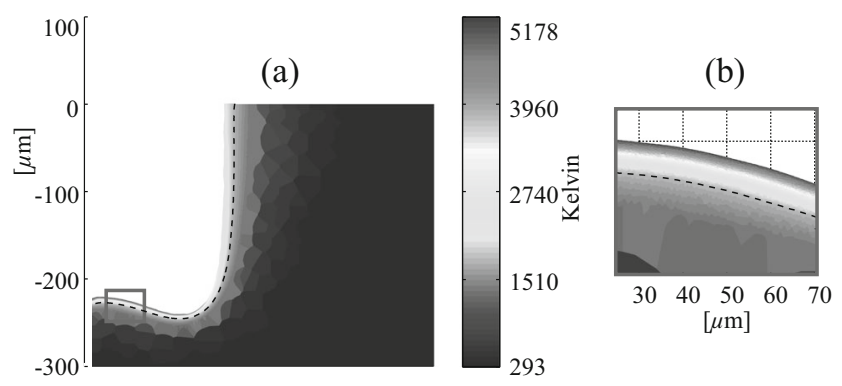

Fig. 16 Temperature field at $150 \mu \mathrm{s}$

The time step is taken equal to $20 \mathrm{~ns}$ and the mesh size criteria in the liquid zone to $0.7 \mu \mathrm{m}$. The computer used to run the simulations is a HP Z800 with a $2.40 \mathrm{GHz}$ and $24 \mathrm{gB}$ RAM double processor "Intel(R) Xeon(R) CPU".

\section{Results}

The total simulation time is about 8 hours. Figure 13 shows the evolution of the number of nodes and the volume of the domain during the simulation. At the beginning, the domain contains 10990 nodes. This number increases as the mesh size criteria induces a strong refinement in the liquid zone, which grows up during the simulation. In the same time, the loss of matter is well observed and the results gives a volume decreasing of $10.5 \%$ at the time $150 \mu$ s.

Figure 14 shows the drilling profile evolution at 50, 100 and $150 \mu \mathrm{s}$ The melted zone is colored in gray. For a better understanding, the laser beam is drawn with a shading representing its spatial shape. The simulation predicts an

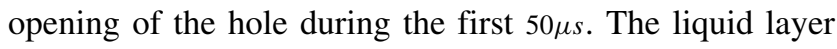
width stays constant after $10 \mu$ s at a value around $10 \mu$ s. The maximum surface temperature is situated at the hole bottom and is about $4800 \mathrm{~K}$. Starting from $100 \mu$ s to $150 \mu$ s, the bottom hole profile is stable and gets deeper with a constant drill velocity at $2.1 \mathrm{~m} / \mathrm{s}$ (Fig. 15a). The hole diameter remains constant and the surface temperature reaches its maximum value at around 5178K (Fig. 15b).

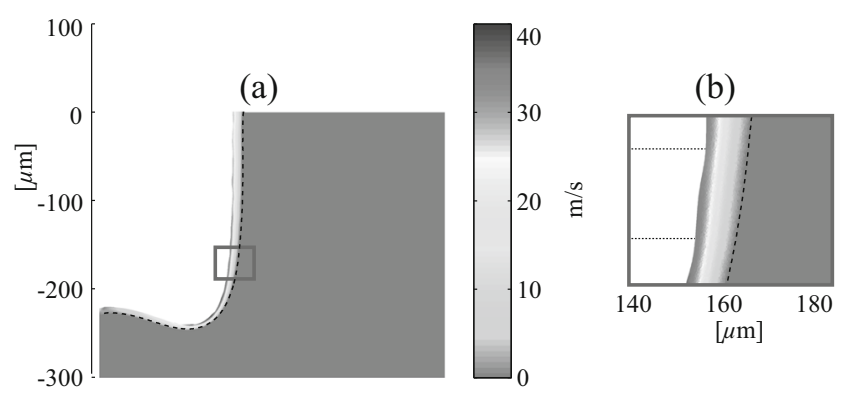

Fig. 17 Velocity magnitude field at $150 \mathrm{~ms}$

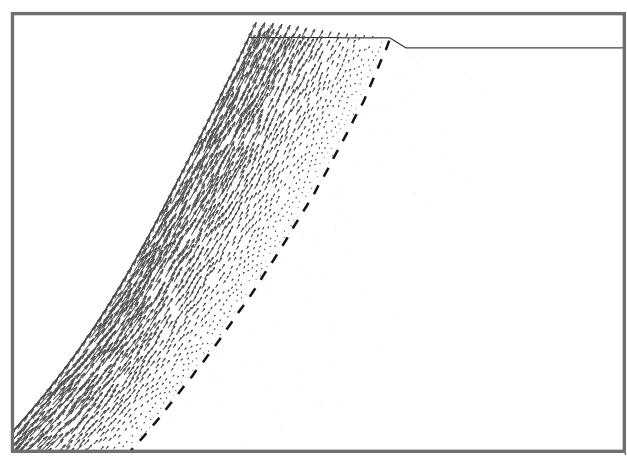

Fig. 18 Velocity field in the liquid at the top of the drill at $50 \mathrm{~ms}$

Figure 16 shows the temperature field in the simulation at $150 \mu \mathrm{s}$. The melting front is drawn with a dashed line. The hottest area during the drilling is situated on the irradiated hole bottom where the maximum temperature reaches $5178 \mathrm{~K}$. The thermal flux induced by the laser aborption is diffused in the liquid and the solid zones with a heat affected zone width from 50 to $100 \mu \mathrm{m}$. The temperature decreases when the liquid climbs up through the hole egdes. The temperature of the hole edge is then around $2500 \mathrm{~K}$.

Figure 17 shows the magnitude of the velocity field computed at $150 \mu$ s and the melting front is always drawn with a dashed line. The simulation describes the moving liquid zone and the unmoving solid one. The maximum velocity is found at the end of the hole edge with a value around 40 $\mathrm{m} / \mathrm{s}$. The flow is almost null at the hole bottom. The viscous effect on the flow is visible on Fig $17 \mathrm{~b}$ with a velocity gradient between the edge and the melting front where the velocity is null. The velocity vectors at the ejection zone of the drilling at time $50 \mu$ s are represented on Fig. 18. The flow follows the curvature of the drilling profile. No instabilities in the flow are noticed, despite the cutting boundary condition on $\partial \Omega_{e}$, which is detailed in the previous "Outflow boundary condition in a Lagrarian description" section.

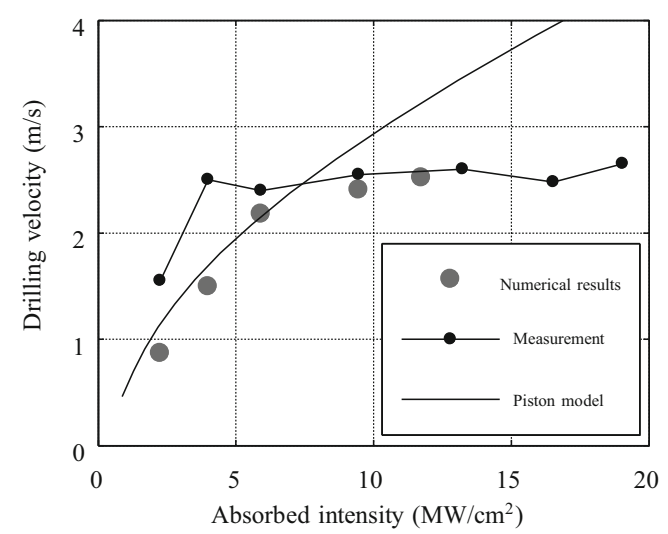

Fig. 19 Drilling velocity for different absorbed intensity 
Fig. $205 \mathrm{~kW}$ and $10 \mathrm{~kW}$ drilling profiles, comparison bewteen the micrographies and the simulation results

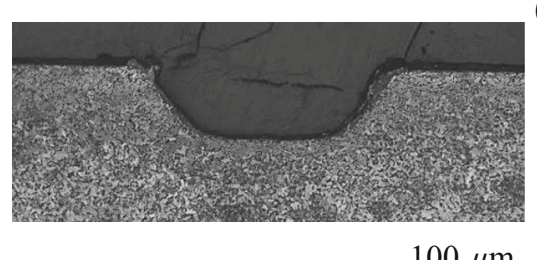

$100 \mu \mathrm{m}$

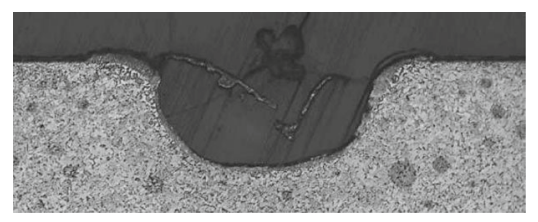

(a)

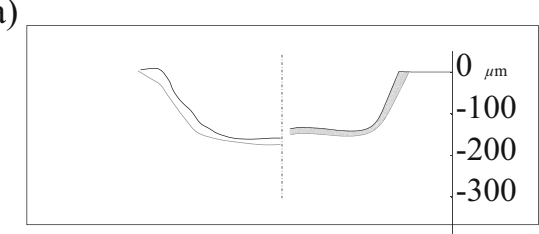

(b)

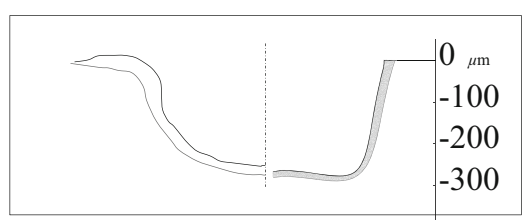

\section{Discussion}

The model developped in this work includes parameters the value of which comes from literature. In other words, any parameter was adjusted to have a good match between experimental and predicted results. The simulation predicts an opening of the hole followed by its enlargement. The enlargment is due to the convective heat transfer carried by the liquid flow.

The quasi-static regime (called percussion) is reached after $100 \mu$ s up to $200 \mu$ s, the end time of the laser pulse. The temperature, flow velocity and drilling velocity remain constant up to the end of the simulation. The predicted liquid zone thickness is about $10 \mu \mathrm{m}$ and the flow velocity at the end of the hole edge is found around $40 \mathrm{~m} / \mathrm{s}$. These values are consistent with experiments in [29].

The drilling velocity can be measured for a range of absorbed intensity from 3 to $13 \mathrm{MW} / \mathrm{cm}^{2}$ [29]. Figure 19 compares the drilling velocity obtained by the measurements, the analytical model (see "Introduction" section ) and the present simulations when the "percussion" regime is reached. According to the experimental results, drilling velocity saturates at $2.5 \mathrm{~m} / \mathrm{s}$ for an absorbed intensity up to $5 \mathrm{MW} / \mathrm{cm}^{2}$. This saturation is well predicted by the simulation while the analytical model predicts a constantly increasing velocity.

To verify that our simulation is able to predict also a hole geometry for low aspect ratio after a non-breaktrough laser drilling, two holes on low-carbon steel were realized with a Trumpf TRUEDISK 10002 laser source. The laser radius was $300 \mu \mathrm{m}$ and two peak powers of $5 \mathrm{~kW}$ and $10 \mathrm{~kW}$ were used. The pulse duration is set to $500 \mu$ s. Simulations are done with these new input parameters. The other ones were unchanged.

Figure 20 shows the cross section at the middle of the holes, experimentaly obtained after laser drilling with $5 \mathrm{~kW}$ peak power (Fig. 20a) and 10kW peak power (Fig. 20b). The hole depth and diameter as well as the resolidifed zone thickness are in good agremment with observations for the two powers.

The enlargement of the hole is, in these simulations, only due to the convective heat transfer carried by the liquid flow. Following discussions in [32], the diameter increase is also due to beam reflexions and radiation heats from the vapor phase, which are not included in our model. Nevertheless, the fact that a good agreement is found between the present simulations and the cross sections can support following knowledges about the laser drilling :

- the vapor phase does not play a significant role for low aspect ratio drilling;

- a quasi-static regime is established after few dozens of microseconds;

- although the convective heat transfer induces a hole enlargement, it seems not sufficient for higher aspect ratio.

\section{Conclusion}

The present work shows numerical simulation of laser drilling in the percussion regime, based on a physical modelling of the process. The model takes into account the absorption of the laser source, the melting of the solid, the energy and mass losses due to vaporization, the vapor pressure which applies on the liquid surface and the resulting liquid flow, the convective and conductive thermal exchanges. These phenomena were already modelled in literature but it is the first time they are all accounted for. The simulations very correctly reproduce the previous phenomena and so the formation of the hole. Using model parameters from literature values and experimental databases and so without using any fit parameters, they predict succesfully the geometry of the hole, the liquid ejection velocity and the drilling velocity at steady state for laser intensities ranging from 3 to $13 \mathrm{MW} / \mathrm{cm}^{2}$. In addition to the inclusion of the key physical events, this sucess comes from the use of the 
original CNEM to solve the 2D axisymetric laser drilling problem which does not need efficient and complex meshing tools as the finite element method. An outflow boundary condition in the Lagragian description was developped to represent the thermal energy and mass losses due to the liquid ejection. Future works head for simulating deeper drilling by increasing the intensity and the pulse duration. Original experimental methods are also in process to measure the surface temperature and the fluid velocity during a laser drilling, in order to compare it with simulation results.

Acknowledgements All the development in this work were based on the Constraint Natural Element Method with the library available at n http://sn-m2p.cnrs.fr/SphinxDoc/cnem/index.html. (ANR)

This work has been supported by the National Research Agency

\section{References}

1. Alaux T, Huchin P, Rosset P, Soulalioux B (2011) Method of manufacturing a turbomachine part comprising cooling air outlets, vol 813. http://www.google.com/patents/EP1813365B1?cl=en, eP Patent 1

2. v Allmen M, Blatter A (1995) Laser-Beam Interactions with Materials: Physical Principles and Applications. Springer Series in Materials Science, Textstream. https://books.google.fr/books? id $=$ wCmEfBF9SewC

3. Anisimov SI (1968) Vaporization of metal absorbing laser radiation. J Exp Theor Phys 27:182

4. Aoki K, Sone Y (1991) Gas flows around the condensed phase with strong evaporation or condensation - fluid dynamic equation and its boundary condition on the interface and their application -. In: Gatignol RS (ed) Advances in Kinetic Theory and Continuum Mechanics. Springer, Berlin, pp 43-54. doi:10.1007/978-3-642-50235-4_5

5. Batteh J, Chen M, Mazumder J (1998) A stagnation flow analysis of keyhole formation in laser drilling. In: ASME heat transfer division, vol 4, pp 29-36

6. Bessagnet F, Commaret P, De S, Hernandez D (2007) Chambre de combustion d'une turbomachine. http://www.google.com/patents/ EP1818613A1?cl=fr. eP Patent App. EP20,070,290,121

7. Braun J, Sambridge M (1995) A numerical method for solving partial differential equations on highly irregular evolving grids. Nature 376:655-660. doi:10.1038/376655a0

8. Chinesta F, Cescotto S, Cueto E. Lorong P (2011) Natural element method for the simulation of structures and processes. WILEY

9. Dausinger F, Shen J (1993) Energy coupling efficiency in laser surface treatment. ISIJ Int 33(9):925-933. doi:10.2355/isijinternational.33.925

10. De Lange DF, Schoonderbeek A, Maijer J (2004) Melt ejection during laser drilling. In: International Congress on Applications of Lasers and Electro-Optics

11. Ge Z, Yu G, He X, Lu G, Li S (2012) Three-dimensional transient numerical simulation of laser drilling process. Scientia Sinica Physica, Mechanica and Astronomica 0, doi:10.1360/132011-1303 http://phys.scichina.com:8083/sciG/ EN/abstract/article_508173.shtml

12. Girardot J, Schneider M, Berthe L, Favier V (2013) Investigation of delamination mechanisms during a laser drilling on a cobaltbase superalloy. J Mater Process Technol 213(10):1682-1691. doi:10.1016/j.jmatprotec.2013.04.003. http://www.sciencedirect. com/science/article/pii/S0924013613001301

13. Gonzáles D, Bel D, Cueto E, Chinesta F, Doblaré M (10) Natural Neighbour Strategies for the Simulation of Laser Surface Coating Processes. Int J Form Process 1:89-108. doi:10.3166/ijfp.10.89-108. http://ijfp.revuesonline.com/article. jsp? articleId $=9631$

14. González D, Cueto E, Chinesta F, Doblaré M (2007) A natural element updated lagrangian strategy for freesurface fluid dynamics. J Comput Phys 223(1):127-150. doi:10.1016/j.jcp.2006.09.002. http://www.sciencedirect.com/ science/article/pii/S0021999106004293

15. Guinard C, Montay G, Guipont V, Jeandin M, Girardot J (2015) Residual stress analysis of laser-drilled thermal barrier coatings involving various bond coats. J Therm Spray Technol 24(12):252-262. http://dx.doi.org/10.1007/s11666-014-0185-z

16. Hirano K, Fabbro R, Muller M (2011) Experimental determination of temperature threshold for melt surface deformation during laser interaction on iron at atmospheric pressure. J Phys D Appl Phys 44(43):435,402-435,412. doi:10.1088/0022-3727/44/43/435402. http://www.ingentaconnect.com/content/iop/jphysd/2011/ 00000044/00000043/art435402

17. Illoul L, Lorong P (2011) On some aspects of the \{CNEM\} implementation in $3 \mathrm{~d}$ in order to simulate high speed machining or shearing. Comput Struct 89(11-12):940-958. doi:10.1016/j.compstruc.2011.01.018. http://www.sciencedirect. com/science/article/pii/S0045794911000307

18. Kamalu J, Byrd P, Pitman A, Variable angle laser drilling of thermal barrier coated nimonic (2002) J Mater Process Technol 122(2-3):355-362. doi:10.1016/S0924-0136(02)00044-4. http:// www.sciencedirect.com/science/article/pii/S0924013602000444

19. Kaminski M (2007) Modélisation de léendommagement en fatigue des superalliages monocristallins pour aubes de turbine en zone de concentration de contrainte. PhD thesis. http://www. theses.fr/2007ENMP1504

20. Ki H, Mohanty PS, Mazumder J (2001) Modelling of highdensity laser-material interaction using fast level set method. J Phys D Appl Phys 34(3):364. http://stacks.iop.org/0022-3727/34/ $\mathrm{i}=3 / \mathrm{a}=320$

21. Knigh0t CJ (1978) Theoretical modeling of rapid surface vaporization with back-pressure. In: 11th Fluid and Plasma Dynamics Conference

22. Knudsen M (1915) Die maximale verdampfungsgeschwindigkeit des quecksilbers. Ann Phys 352(13):697-708. doi:10.1002/andp.19153521306

23. Low DKY, Li L, Byrd PJ (2002) Hydrodynamic physical modeling of laser drilling. J Manuf Sci Eng 124(4):852-862. http://cat. inist.fr/?aModele $=$ afficheN\&cpsidt $=14014282$

24. Martin JC, Moyce WJ (1952) Part iv. an experimental study of the collapse of liquid columns on a rigid horizontal plane. Philos Trans R Soc A Math Phys Eng Sci 244(882):312-324. doi:10.1098/rsta.1952.0006

25. Mills K (2002) Recommended Values of Thermophysical Properties for Selected Commercial Alloys. Woodhead Publishing Series in Metals and Surface Engineering Series, Woodhead. https:// books.google.fr/books? $\mathrm{id}=\mathrm{GaOooR} 231 \mathrm{aAC}$

26. Revuz N (2010) Etude de l'endommagement d'un superalliage monocristallin base de nickel induit par percage laser. Engineering school of Mines ParisTech, $\mathrm{PhD}$ thesis

27. Samokhin AA (1988) First Order Phase Transitions Induced by Laser Radiation in Absorbing Condensed Matter. Effect of Laser Radiation on Absorbing Condensed Media. In: Nauka, Moscowpp, 1

28. Schneider M (2006) Perçage profond par laser: analyse des processus physiques. Pierre et Marie Curie University, $\mathrm{PhD}$ thesis 
29. Schneider M, Muller M, Fabbro R, Berthe L (2007) Study of hole properties in percussion regime with a new analysis method. J Laser Micro/Nanoeng 2(2):128-132. doi:10.2961/jlmn.2007.02.0004

30. Schneider M, Berthe L, Fabbro R, Muller M (2008) Measurement of laser absorptivity for operating parameters characteristic of laser drilling regime. J Phys D Appl Phys 41(15):155,502. http:// stacks.iop.org/0022-3727/41/i=15/a=155502

31. Schneider M, Girardot J, Berthe L (2011) Recoil Pressure and Surface Temperature in Laser Drilling. In: International Congress on Applications of Lasers and Electro?-Optics

32. Schulz W, Eppelt U, Poprawe R (2013) Review on laser drilling I. Fundamentals, modeling, and simulation. Journal of Laser Applications 25(1):012,006. doi:10.2351/1.4773837. http://scitation. aip.org/content/lia/journal/jla/25/1/10.2351/1.4773837

33. Semak V, Matsunawa A 1997-12-01T00:00:00 The role of recoil pressure in energy balance during laser materials processing. $\mathrm{J}$ Phys D Appl Phys 30(18):2541-2552. http://www.ingentaconnect. com/content/iop/jphysd/1997/00000030/00000018/art00008
34. Sukumar N, Moran B, Belytschko T (1998) The natural element method in solid mechanics. Int J Numer Methods Eng 43(5):839887. http://dx.doi.org/10.1002/(SICI)1097-0207(19981115)43: 5<839::AID-NME423〉3.0.CO;2-R

35. Traversoni L (1994) Natural neighbor finite elements. In: International Conference on Hydraulic Engineering Software

36. Ytrehus T, Østmo S (1996) Kinetic theory approach to interphase processes. Int $\mathrm{J}$ Multiphase Flow 22(1):133-155. doi:10.1016/0301-9322(95)00056-9. http://www.sciencedirect. com/science/article/pii/0301932295000569

37. Yvonnet J, Ryckelynck D, Lorong P, Chinesta F (2004) A new extension of the natural element method for non-convex and discontinuous problems: the constrained natural element method (c-nem). Int J Numer Methods Eng 60(8):1451-1474. doi:10.1002/nme.1016

38. Yvonnet J, Chinesta F, Lorong P, Ryckelynck D (2005) The constrained natural element method (c-nem) for treating thermal models involving moving interfaces. Int J Therm Sci 44(6):559-569. doi:10.1016/j.ijthermalsci.2004.12.007. http://www.sciencedirect. com/science/article/pii/S1290072905000347 